\title{
FPGA-Based Voltage Measurements in AC Drives
}

\author{
Luca Peretti, Mauro Zigliotto
}

\begin{abstract}
In the modern sensorless and self-commissioning drives scenario, the availability of precise phase voltage measurement represents an undoubted advantage. But the task is not trivial, and few viable solutions are available. The present paper proposes an up-to-date digital method for the measurement of the instantaneous phase voltages of a three-phase PWM inverter. The phase-to-phase PWM inverter voltages are oversampled with respect to the switching period by fast $A / D$ converters, which feed the FPGA-based digital integrator. The digital output is ready for further uses in any advanced control and estimation algorithm. Measurement repeatability, reduced size, tuning-free circuit are among the inherent side-advantages of the FPGAbased solution with respect to the existing analog ones. The paper includes design hints and a full batch of experimental tests and validations.
\end{abstract}

Index Terms-AC motor drives, Field programmable gate arrays, Sensorless control, Voltage measurement.

\section{INTRODUCTION}

$\mathbf{T}$ HE trend of modern (sensorless) electrical drives is toward the elimination of every transducer which comprises moving parts. This yields remarkable savings in production, installation and maintenance, as well as improved reliability. Generally, in sensorless drives the essential measurements of speed and/or position are replaced by mathematical models, which are fed by voltage and current measurements. While the latter is anyway available for both current control and diagnostics, the voltage measurement is still an open issue. Very often, in the (even recent) past, the solution has been shifted to the seeking of the best voltage compensation strategy ([1], [2]). Actually, in a perfectly compensated voltage inverter, the references can be profitably used instead of the actual quantities. Anyway, the causes of inverter non-linearity are manifold and, very often, they are device-dependent. The worst effects of the blanking times inserted between the commutation of the switching devices of the same inverter leg is experienced at low phase voltages, which normally corresponds to the low speed region, in which the availability of reliable voltage measurement is of the utmost importance in every sensorless scheme. Moreover, the inverter voltage non-linearity compensation deals with a precise detection of current zero-crossings, which often calls for particular care and extra-hardware wiring to cope with disturbances elimination. A different approach is the direct measurement of the inverter voltages. Surely, in the market are present voltage sensors, which are based on the same current sensors principle. But they are quite expensive, and, normally, their bandwidth cannot deal with the high frequency nature of the PWM phase voltages. The measurement of the instantaneous average value of the output

Luca Peretti and Mauro Zigliotto are with the Department of Technique and Management of Industrial Systems of the University of Padova, Stradella San Nicola 3, 36100 Vicenza, Italy (e-mail: luca.peretti@unipd.it, mauro.zigliotto@unipd.it). voltage during each PWM switching period could represent a great improvement for every sensorless scheme, or in the case where on-line parameter identification is going to be undertaken. Yet, the measurement is rather troublesome and in the literature there exist only few examples. One of the first examples is reported in [3], and a simplified version is proposed in [4]. In both cases, the direct measure within each PWM switching period is performed without any lowpass filtering action, whose related phase-shift could lead to performance degradation in sensorless applications. Anyway, they both foresee an analog integral action, whose accuracy may be corrupted by components tolerance and ageing. The present paper proposes a up-to-date digital method for the measurement of the instantaneous phase voltages of a threephase PWM inverter. The pseudo-square wave voltages are oversampled with respect to the switching period by fast A/D converters, which feed the FPGA-based integrator. The output, already in the digital form, is ready for further uses in any digital sensorless algorithm, including a FPGA-based drive system. Measurement repeatability, fixed accuracy and excellent linearity are among the inherent side-advantages of the proposed technique with respect to the existing analog solutions.

The paper is organised as follows. Sect. II recalls the most known compensation strategies, which are adopted to estimate the phase voltages from the reference signals issued by the control algorithms. Sect. III focuses on the direct PWM voltage measurement in sensorless electrical drives. Sect. III-A briefly recalls the existing analog solution for phase-to-phase voltage measurement, while Sect. III-B presents the digital equivalent scheme based on a FPGA board. The FPGAbased solution is then discussed into details, including signal conditioning and digital requirements. Sect. IV includes design hints and a full batch of experimental tests and validations.

\section{Voltage COMPEnSATION STRATEGIES}

Compensation strategies for inverter non-linearities aims to get a quasi-perfect match between reference and actual motor phase voltages.

Compensation strategies can be applied in different reference frames - the three-phase $a b c$, the stationary $\alpha \beta$ and the rotating $d q$ reference frame. Compensations in the $a b c$ frame refer to the conventional theory of dead-time effects. They cope with the average voltage reduction per carrier period in an inverter leg, by adding in advance an opposite quantity to the reference voltages. Fig. 1 shows the typical voltage output of an inverter leg with dead times, within each switching period $T_{c}$. It is:

$$
T_{a, O N}=T_{a, O N}^{*}-t_{d} \operatorname{sgn}\left(i_{s a}\right)
$$


where $T_{a, O N}^{*}$ is the commanded switch-on time for the upper IGBT, $T_{a, O N}$ is the actual one, $t_{d}$ is the dead time and sgn is the sign function.
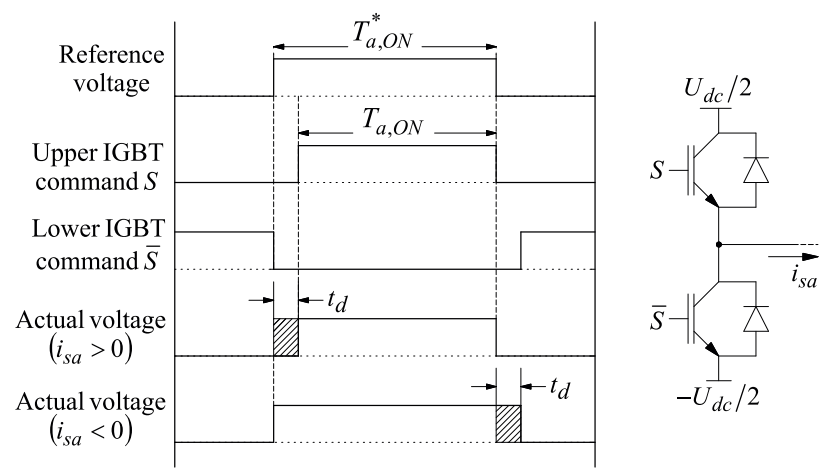

Fig. 1. Voltage output of an inverter leg with dead times.

The mean value of the voltages, referred to the DC bus midpoint $n$, is:

$$
u_{\text {san }}=u_{\text {san }}^{*}-\frac{t_{d}}{T_{c}} U_{d c} \operatorname{sgn}\left(i_{s a}\right)=u_{\text {san }}^{*}-u_{d i s t, a n}
$$

where $u_{\text {san }}^{*}$ is the reference voltage, $u_{\text {san }}$ is the actual one, $U_{d c}$ is the DC bus voltage. The conventional dead-time compensation in the $a b c$ frame adds the term $u_{\text {dist, an }}$ to the reference voltage $u_{\text {san }}^{*}$, and the same applies to the other inverter legs. The solution suffers of chattering at low current levels, because of the difficult sign detection. Moreover, the strategy does not account for other practical issues, as the finite switch-on and switch-off times of the IGBTs, the on-state voltage drop and other parasitic effects. A more precise compensation should include these effects, as shown in [5].

Compensations in the $\alpha \beta$ frame transform the (2) in the stationary reference frame, thus having the same problems of the $a b c$-frame-based solutions. A bright solution, which takes into account IGBTs voltage drops and diode forward voltage, is presented in [1].

The last group of compensation techniques operate in the $d q$ reference frame, fixed to the current phase. In this frame, the phase voltage distortions (2) can be expressed as the sum of multiple of sixth harmonics plus a constant term. The problem of the detection of the current sign is shifted to the knowledge of the current angle, and the perfect disturbance rejection of repetitive controllers can be profitably exploited, as in [2].

\section{Voltage MEASUREMENT IN AC DRIVES}

A different approach to the knowledge of the phase voltages is based on the real-time measurement of their values. Actually, the measurement does not substitute the compensation algorithms mentioned earlier in Sect. II. A measurement of the phase voltage could rather fix any remaining uncertainty that could come from an imperfect compensation. A general block schematic of a PWM voltage measurement solution is shown in Fig. 2.

The fast control prototyping (FCP) board is responsible for both the current and speed control loops of a conventional FOC drive. The dashed line on the $i_{c}$ current of Fig. 2 indicates

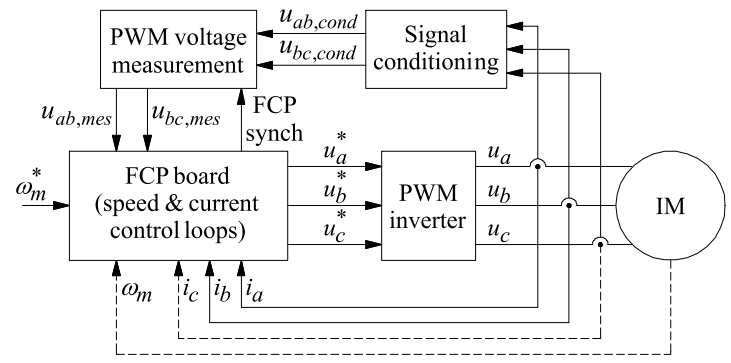

Fig. 2. Block schematic of the IM drive with PWM voltage measurement.

that the signal sampling could be omitted, in case of insulated neutral point. Similarly, the dashed line on the mechanical speed $\omega_{m}$ recalls that the drive could be in sensorless mode.

Parallel to the drive, a signal conditioning block and a PWM voltage measurement block process the phase voltages and provide a measurement of the phase-to-phase voltages to the drive. The former reduces the phase voltage value within the input range of the $\mathrm{A} / \mathrm{D}$ measurement circuit, while the latter performs the measure.

The phase voltages on the motor side are pseudo-square waves, and just the mean values within each PWM cycle are of interest. Such operation requires a line provided by the FCP (FCP synch) for the tight synchronisation with the PWM signal, as shown in Fig. 2.

The PWM voltage measurement block can be implemented in either analog or digital way. The analog solution, formerly adopted also by other Authors (see [3], [4]), is briefly reported hereafter, while the rest of the paper will focus on the proposed digital solution.

\section{A. Analog solution}

The analog measurement block consists of two operational amplifiers in the integrator configuration, connected in parallel [6]. The principle of operation is recalled in Fig. 3 for the phase-to-phase voltage $u_{a b}$, and the same holds for $u_{b c}$.

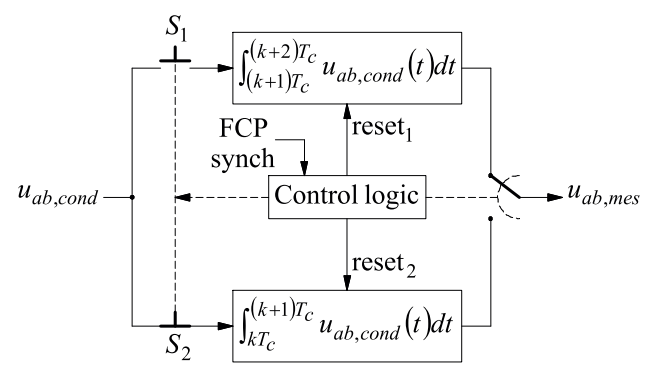

Fig. 3. Block schematic of the analog PWM voltage measurement block.

The FCP system provides a synchronisation signal (FCP synch in Fig. 3), at a rate equal to the PWM switching frequency $\left(1 / T_{c}\right)$. A sequential logic manages the input to the integrators through two DG611 Hi-Speed, Low-Glitch D/DMOS Analog Switches $S_{1}$ and $S_{2}$. Each integrator returns the mean value of the voltage within a switching period, in a complementary way. That is, while one integrator is working the other is reset, and its output is tied up to the output $u_{a b, m i s}$. The use of integrators in parallel enables a zero-time delay 
between the actual voltage and the measured one, which is a key-point for the accuracy of the measurement. Actually, it can be easily understood that with a single integration path, there would be no time left to reset the output to zero at the beginning of the next PWM period, without losing part of the signal $u_{a b, c o n d}$.

High bandwidth, high slew-rate switches and op amps have been used in order to assure the accuracy of the integration. The passive elements should be manually matched to improve the overall precision, since the two integrators must be identical. Some circuital solutions, as self-compensating topologies, could also guarantee a low sensitivity to temperature variations. For these reasons, the analog-based PWM voltage measurement is meant for custom applications, as research labs, rather than for larger production.

\section{B. FPGA-based digital solution}

Gathering the know-how gained during the experiments with the analog solution of Sect. III-A, a PWM voltage measurement based on a FPGA has been developed and tested.

The selected FPGA board was an Altera Cyclone III EP3C25, with 32-MB DDR SDRAM, a 16-MB Flash memory, a 1-MB high-speed SSRAM, 25000 programmable logic blocks and 0,6 MB of memory blocks. The main board was connected to a daughter board equipped with two A/D converters for phase-to-phase voltage acquisition, one input for the PWM synchronisation, and two D/A converters to provide the measured phase-to-phase voltages to the FCP board. Of course, they could be omitted in industry applications, where the digital measurement of the voltage is directly connected to the control microprocessor. The A/D and the D/A converters have a resolution of 14 bits, working at a sample frequency between $1 \mathrm{MHz}$ and $65 \mathrm{MHz}$. Both converter types are from Analog Devices, respectively AD9248 for the A/D device and AD9767 for the D/A device. It is worth to note that the board exceeds by far the requirements and, as many research equipments do, it well fits for fast prototyping, rather than for cost- or volume-related issues.

An insight of the signal conditioning block of Fig. 2 is shown in Fig. 4.

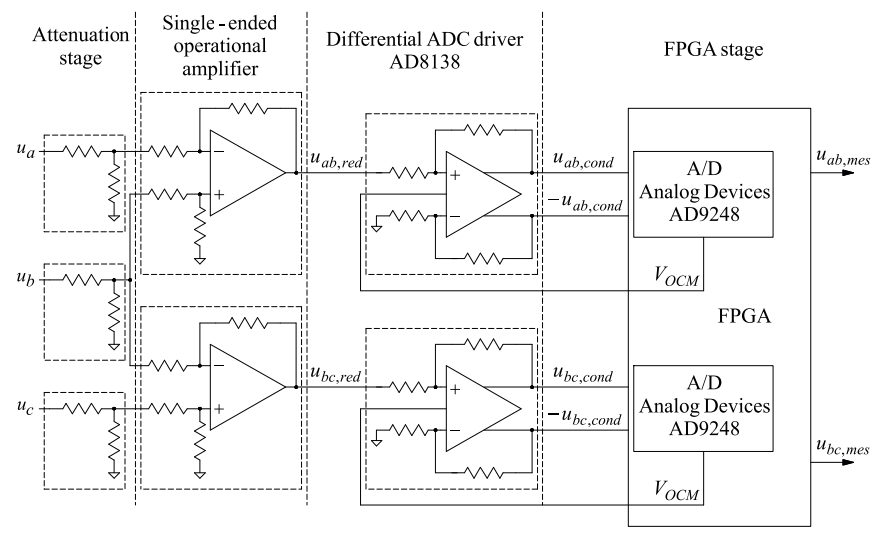

Fig. 4. Signal conditioning block for the FPGA-based measurements.

The signal conditioning is performed by three cascaded sections. The first one is a ratiometric measurement of the amplitudes of the actual phase voltages, which feed the second stage, composed by two single-ended operational amplifiers for impedance matching.

The A/D converter features the best signal-to-noise ratio when fed by differential input. Therefore, the last section comprises two differential drivers (Analog Devices AD8138). The common-mode voltage $V_{O C M}$ used to bias the differential input is provided by the A/D converter itself, and fed back to the differential driver. The obtained signals $u_{a b, c o n d}$, $-u_{a b, c o n d}, u_{b c, \text { cond }}$ and $-u_{b c, c o n d}$, biased by $V_{O C M}$, are the input of the A/D converter. In this work, $V_{O C M}$ was fixed at $1,65 \mathrm{~V}$, half of the DC supply of 3,3 V.

Further to the improvement of the signal-to-noise ratio, the use of a differential driver yields other benefits in terms of signal integrity. Differential-mode signals could be equivalently obtained by means of three-taps transformers with the central tap connected to the zero voltage reference. However, commercial transformers for PCBs are usually suitable for higher frequencies, in the order of $\mathrm{MHz}$ or tens of $\mathrm{MHz}$, while conventional frequencies for PWM voltage inverters are about tens of $\mathrm{kHz}$. Moreover, the DC value of the voltage is lost when a transformer is used, while it is maintained with the differential driver.

The gain of the third stage was designed to get the widest full-scale value. In particular, the maximum input voltage of the A/D converter corresponds to a maximum phase-to-phase voltage of $\pm 300 \mathrm{~V}$.

The signals $u_{a b, c o n d}$ and $u_{b c, c o n d}$ are fed to the PWM voltage measurement block. Fig. 5 shows the block scheme of the measurement of one phase-to-phase voltage (the second one being identical).

At a first glance, the scheme of Fig. 5 resembles the twointegrators method explained in Sect.III-A. As a matter of fact, the scheme does translate the analog solution into the FPGA board, but with distinctive advantages over the problems caused by the analog implementation.

As a first step, the differential signals are sampled by the A/D converter and processed to transform them into a two's complement representation. The 14 bits signals are cast to a 27-bit representation, suitable for the integral accumulators. The choice of 21 bits derives from a worst-case scenario, where the sampled signal is equal to its maximum value (13 bits equal to 1 , the last bit represents the sign) for a whole PWM period. Since the accumulators, as the A/D converters, operate at a clock frequency of 1,28 MHz, and supposing a switching frequency of $10 \mathrm{kHz}$, a PWM period would allocate $(1,28 \mathrm{MHz}) /(10 \mathrm{kHz})=128$ samples. Thus, the maximum value of the integral sum would be

$$
\frac{1,28 \mathrm{MHz}}{10 \mathrm{kHz}}\left(2^{13}-1\right)=1048448
$$

which is representable with 20 bits, plus 1 bit for the sign.

The two's complement representation for the sampled voltages eases the integral sum, which is automatic. In offset binary mode, negative and positive values should have been detected apart, before deciding for a sum or a subtraction.

As shown in Fig. 5, accumulators are reset via a synchronisation signal (FF synch) which is related to the synchronisation 


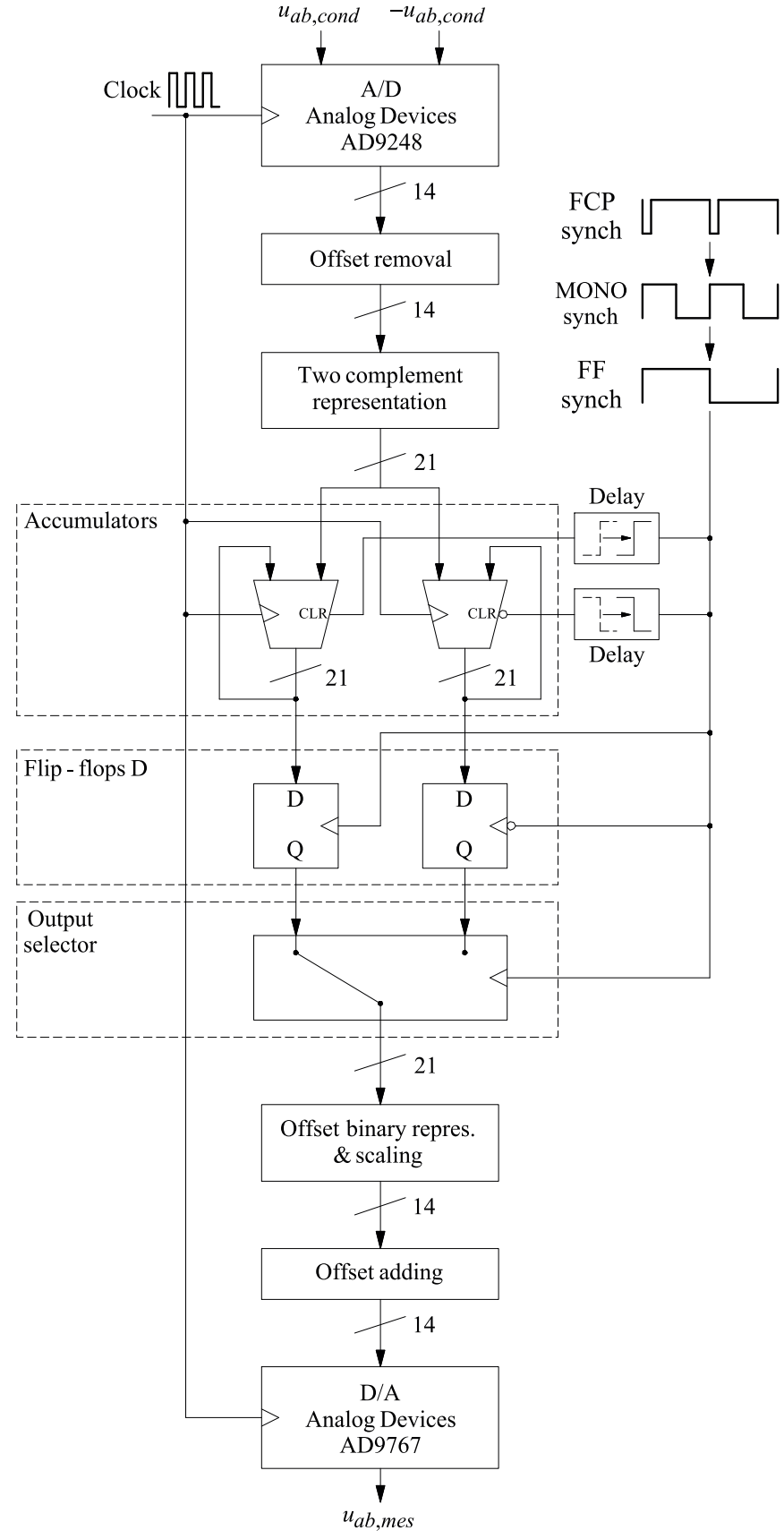

Fig. 5. Block schematic of the FPGA-based voltage measurement circuit.

provided by the FCP board (FCP synch). The aim of the FF synch is to let the accumulators work in a complementary way over an entire PWM period. Thus, the FF line toggles between logical high and low each PWM period, resulting in a squarewave signal at half of the PWM frequency. Fig. 6 shows how the FF synch is obtained from the FCP synch.

The FCP synch is generated by the built-in PWM generator inside the voltage inverter. It features an asymmetric square-wave signal with a very narrow low pulse at each new period starting. The pulse signal is enlarged and stabilysed by a monostable multivibrator (National Semiconductor CD4538B), which generates an intermediate square wave (MONO synch).



Fig. 6. Generation of the FF synch signal for the FPGA board.

The double-period FF synch signal is then obtained from MONO synch by means of a JK flip-flop. The right and left accumulators in Fig. 5 are level-driven by the FF synch line.

As stated before, the accumulators work in a complementary way. While one is adding the voltage samples originated from the A/D conversion, the other accumulator is reset and its output kept to zero.

The two D-type flip-flops sample and maintain the integral output of their correspondent accumulator for an entire PWM period. It is clear that the input of a flip-flop should be sampled exactly when a rising or falling edge of FF synch occurs (depending on the considered flip-flop). A concurrent activation of the CLR signal for the corresponding accumulator, by means of the same FF synch, could potentially cause metastability problems: the accumulator would be reset and the flip-flop D would sample the input at the same interval. The problem is solved by the introduction of a small delay in the CLR signal path, in order to reset the accumulator slightly after the flipflop D has sampled its input. The delay only affects the rising edge of the CLR input in the left accumulator, and the falling edge of the CLR input in the right accumulator as well.

Considering the system as a whole, when FF synch is logical high the right accumulator is integrating, while the left one is reset. The output selector of Fig. 5 chooses the left flip-flop D as output, since it is maintaining the value of the integration over the previous PWM period. When a high-to-low transition of the FF synch signal occurs, the input of the right flip-flop $\mathrm{D}$ is sampled and brought as the updated output, while the left accumulator starts to integrate the voltage over a new PWM period. As said, after a small delay the right accumulator is reset, waiting for the next edge of the FF synch to occur.

The last stage of the measurement circuit transforms the two's complement output into an offset binary representation, and shifts them to 14 bits, in order to fulfil the requirements of the D/A converter. It is worth to note that this step is done for evaluation purposes only, since the two's complement digital output already represents the required voltage measurement, which could be directly integrated into the rest of the drive.

\section{EXPERIMENTAL RESULTS}

The FPGA-based solution was tested on an experimental test-bench. A conventional three-phase inverter was connected to a $3 \mathrm{~kW}-1500 \mathrm{rpm}$ induction motor, with $20 \mathrm{Nm}$ of nominal torque, 2,6 Arms of nominal current and two pole pairs. The 
three-phase voltages were brought to the motor and to the signal conditioning block, as shown in Fig. 2.

The motor and the inverter were controlled by means of a FCP board, which provided the synchronisation signal for the monostable multivibrator. The motor was controlled by a simple $\mathrm{V} / \mathrm{Hz}$ program without dead time compensation, in order to emphasize the voltage distortions caused by dead times. The PWM frequency and the current sample frequency were of $10 \mathrm{kHz}$, while the DC bus voltage was set to $200 \mathrm{~V}$. The FPGA sampling frequency was set to 1,28 $\mathrm{MHz}$.

Fig. 7 reports a measurement of the mean value of $u_{a b}$ when the motor was rotating at $50 \mathrm{~Hz}$, with a reference phase voltage amplitude of $70 \mathrm{~V}$ (phase-to-phase voltage of $121 \mathrm{~V}$ ). The plot shows the phase-to-phase voltage $u_{a b}$, its actual mean value and the measurement obtained from the FPGA. Fig. 8 reports the percentage error between the actual mean value of $u_{a b}$ and the measured one, normalised to the maximum amplitude of a sinusoidal voltage given the DC bus voltage $\left(U_{N}=200 / \sqrt{3}\right.$ $\mathrm{V})$.

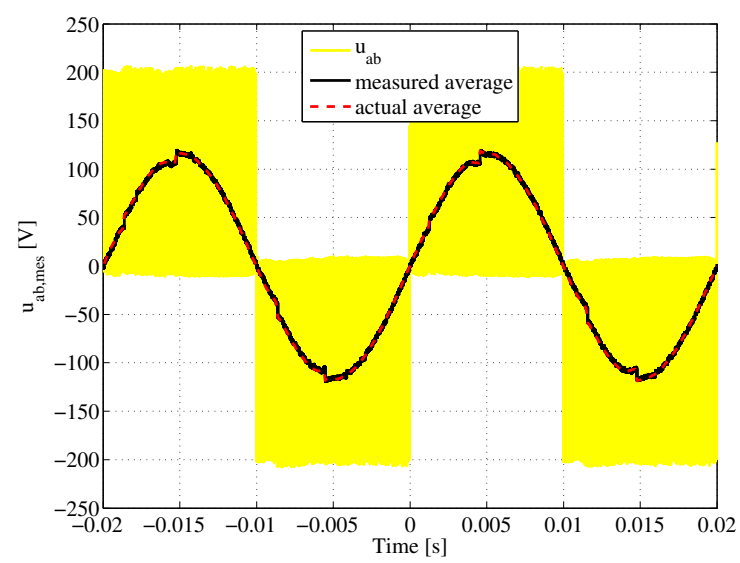

Fig. 7. $u_{a b}$ measure with FPGA sampling rate of $1,28 \mathrm{MHz}$.

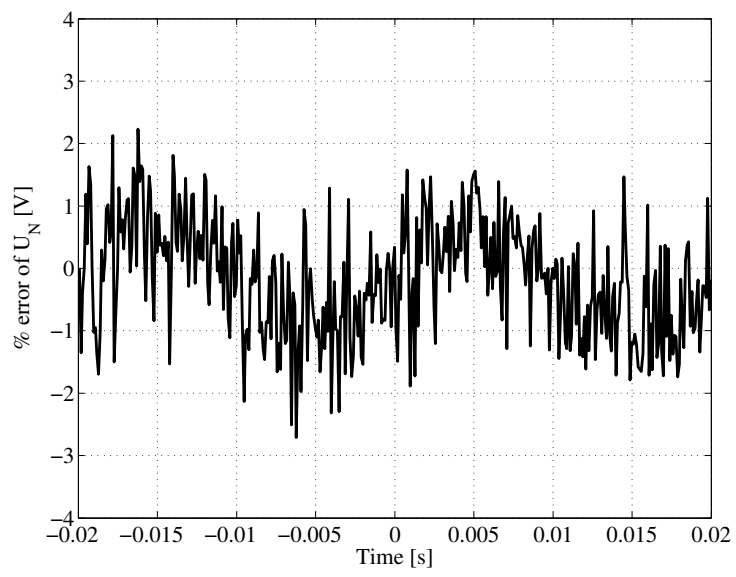

Fig. 8. Percentage error on $u_{a b}$ measure (FPGA @ 1,28 MHz).

The actual mean value of $u_{a b}$ was obtained by means of an off-line post-processing of the data. From the $u_{a b}$ samples and the PWM synchronisation signal provided by the FCP board, an off-line integrator was performed, integrating $u_{a b}$ for each PWM period to resemble the FPGA-based algorithm.
The measured mean value was multiplied by its attenuation factor, in order to compare the measurements. Results show a very good agreement between the actual value and the measured one, including when the phase-to-phase voltage abruptly changes due to the dead-time voltage distortion, which is dependant on the sign of the currents.

The same operating conditions were maintained, while the FPGA sampling rate was set to $20 \mathrm{MHz}$. Results of the average voltage measurement and the percentage error are shown in Fig. 9 and Fig. 10.



Fig. 9. $u_{a b}$ measure with FPGA sampling rate of $20 \mathrm{MHz}$.



Fig. 10. Percentage error on $u_{a b}$ measure (FPGA @ $20 \mathrm{MHz}$ ).

The increase of the FPGA sampling frequency did not lead to a proportional increase of performances. It was found that the percentage error at $20 \mathrm{MHz}$ was half of that at $1,28 \mathrm{MHz}$, but still the latter was low enough to justify the exploitation of a low-cost A/D converter, suitable for largevolume productions.

The measurement was repeated by changing the motor to a surface permanent magnet one (nominal speed $3000 \mathrm{rpm}$, nominal torque $14,1 \mathrm{Nm}$ ). The PWM sampling time was increased to $15 \mathrm{kHz}$ while maintaining the FPGA sampling frequency at $1,28 \mathrm{MHz}$ and the DC bus at $200 \mathrm{~V}$. The motor was rotating with an open loop algorithm at a frequency of 5 $\mathrm{Hz}$ and a phase voltage amplitude of $15 \mathrm{~V}$, without dead time compensation. Results are shown in Fig. 11 Fig. 12. 


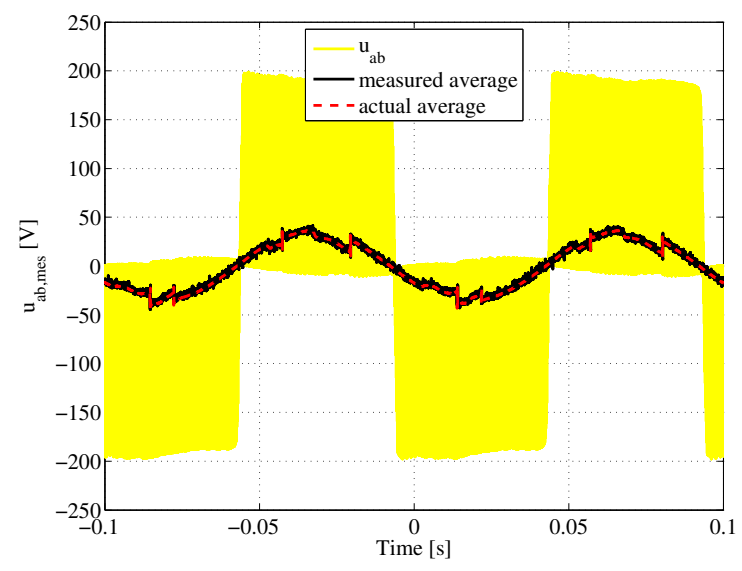

Fig. 11. $u_{a b}$ measure with FPGA sampling rate of 1,28 MHz (SPM).

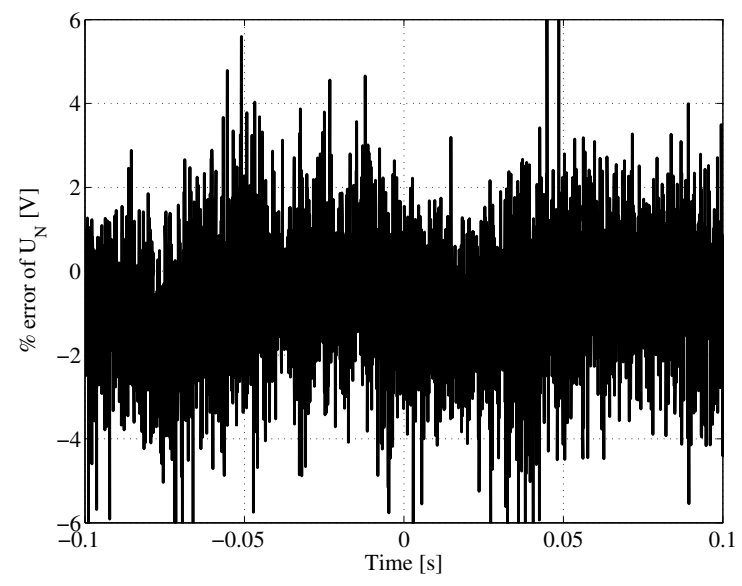

Fig. 12. Percentage error on $u_{a b}$ measure (SPM, FPGA @ 1,28 MHz).

Results show that the increase of the PWM frequency, while maintaining the FPGA sampling frequency, led to increased errors, but still within acceptable values.

The input-output linearity of the FPGA-based solution was also investigated by feeding the measurement system with DC voltage values obtained directly from the DC bus. At 1,28 $\mathrm{MHz}$ and considering a PWM frequency of $10 \mathrm{kHz}$, the system proved to be very linear over the entire range of measure, with a mean attenuation of 616,13, (standard deviation $\sigma=$ 0,47 , a maximum percentage error of $0,17 \%$ and an average percentage error of $0,0281 \%$.

Tab. I reports the FPGA logic port requirements of the proposed solution. As expected, the total occupancy area of the proposed solution is rather small with respect to the available logic ports and registers.

Tab. I

LOGIC REQUIREMENTS FOR THE FPGA-BASED MEASUREMENT SOLUTION.

\begin{tabular}{c|cc}
\hline Total logic elements & $1295 / 24624$ & $(5 \%)$ \\
\hline Dedicated logic registers & $1012 / 24624$ & $(4 \%)$ \\
\hline Total PLLs & $1 / 4$ & $(25 \%)$
\end{tabular}

\section{CONCLUSIONS}

A digital solution for the direct measurement of the motor phase voltages in AC drives was presented. The technique consists of a fast A/D oversampling of the voltage, followed by a digital FPGA-based integration. The output is the mean value of the voltage over each PWM period. The obtained measurements could be of great utility in sensorless speed estimation algorithms, as well as in on- and off-line parameter estimation for the next generation of self-commissioning drives. Experimental tests and extensive batch of measurements proved the effectiveness of the solution, showing also its reduced implementation complexity.

\section{ACKNOWLEDGEMENTS}

Authors would like to thank REEL s.r.l. (Ponte di Nanto, Italy), Mr. C. Pomari and Mr. P. Graffagnino for their help and support during the experimental stage of this work.

\section{REFERENCES}

[1] J.-W. Choi and S.-K. Sul, "Inverter output voltage synthesis using novel dead time compensation," IEEE Trans. Power Electron., vol. 11, no. 2, pp. 221-227, Mar. 1996.

[2] S. Bolognani, L. Peretti, and M. Zigliotto, "Repetitive-control-based selfcommissioning procedure for inverter non-idealities compensation," IEEE Trans. Ind. Appl., vol. 44, no. 5, pp. 1587-1596, Sep./Oct. 2008.

[3] T.-H. Chin, M. Nakano, and T. Hirayama, "Accurate measurement of instantaneous voltage for power electronics circuits," in Proceedings of Power Conversion Conference, PCC'97, vol. 2, Nagaoka, Japan, Aug. 1997, pp. 881-884.

[4] T. Batzel and M. Comanescu, "Instantaneous voltage measurement technique for PWM voltage source inverters," International Journal on Power Electronics, vol. 1, no. 1, pp. 1-16, 2008.

[5] A. R. Muñoz and T. A. Lipo, "On-line dead-time compensation technique for open-loop PWM-VSI drives," IEEE Trans. Power Electron., vol. 14, no. 4, pp. 683-689, Jul. 1999.

[6] S. Bolognani, L. Peretti, M. Zigliotto, and E. Bertotto, "Commissioning of electromechanical conversion models for high dynamic PMSM drives," IEEE Trans. Ind. Electron., vol. 57, no. 3, pp. 986-993, Mar. 2010.

Luca Peretti received the M. Sc. degree in electronic engineering from the University of Udine, Italy, in 2005, and the Ph.D. in mechatronics and industrial systems from the University of Padova, Italy, in 2009. From November 2007 to March 2008 he has been a visiting Ph.D. student at ABB Corporate Research Center, Department of Power Technologies, Västerås, Sweden. From January 2009 he is helding a post-doctoral research position at the Department of Technique and Management of Industrial Systems, University of Padova, Vicenza, Italy. His main research activity concerns advanced sensorless control and parameter estimation techniques for electrical motor drives.

Mauro Zigliotto received the Laurea degree in electronic engineering from the University of Padova, Padova, Italy, in 1988. He worked in industry as an R\&D Manager, developing DSP-based control systems for electric drives. From 1992 to 1999 , he was a Senior Research Assistant with the Electric Drives Laboratory, University of Padova. In 2000, he joined the Department of Electrical, Management and Mechanical Engineering, University of Udine, Udine, Italy, as an Associate Professor of electric drives. Since November 2005, he has been with the Department of Technique and Management of Industrial Systems, University of Padova, Vicenza, Italy, where he started working in the Electric Drives Laboratory. His main research interests include advanced control strategies for AC motors, and he has published extensively in this area. Prof. Zigliotto is currently the Secretary of the IEEE IAS/IES/PELS North Italy Joint Chapter. He has been serving international conferences as a member of the Steering or Technical Committees. 